\title{
Revealing and concealing entanglement with noninertial motion
}

\author{
Marko Toroš $\odot,{ }^{1, *}$ Sara Restuccia, ${ }^{2}$ Graham M. Gibson, ${ }^{2}$ Marion Cromb $\odot,{ }^{2}$ Hendrik Ulbricht, ${ }^{3}$ \\ Miles Padgett, ${ }^{2}$ and Daniele Faccio ${ }^{2, \dagger}$ \\ ${ }^{1}$ Department of Physics and Astronomy, University College London, Gower Street, London WC1E 6BT, United Kingdom \\ ${ }^{2}$ School of Physics and Astronomy, University of Glasgow, Glasgow G12 8QQ, United Kingdom \\ ${ }^{3}$ Department of Physics and Astronomy, University of Southampton, Southampton SO17 1BJ, United Kingdom
}

(Received 14 November 2019; revised manuscript received 24 March 2020; accepted 30 March 2020; published 27 April 2020)

\begin{abstract}
Photon interference and bunching are widely studied quantum effects that have also been proposed for high-precision measurements. Here, we construct a theoretical description of photon interferometry on rotating platforms, specifically exploring the relation between noninertial motion, relativity, and quantum mechanics. On the basis of this, we then propose an experiment where photon entanglement can be revealed or concealed solely by controlling the rotational motion of an interferometer, thus providing a route towards studies at the boundary between quantum mechanics and relativity.
\end{abstract}

DOI: 10.1103/PhysRevA.101.043837

\section{INTRODUCTION}

The notions of space and time are at the core of modern physics and remain an area of intense research [1,2]. A striking example of how elementary notions of space and time lead to surprising consequences is the derivation of Lorentz transformations, a cornerstone of quantum field theory, utilizing only basic assumptions $[3,4]$.

The exploration of the special-relativistic regime is historically strongly linked to investigations of the propagation of light [5], e.g., the Michelson-Morley experiment [6]. More recent experiments have also started to probe the quantum nature of light, e.g., the Hong-Ou-Mandel (HOM) experiment [7], indirectly testing the underpinning spacetime symmetries. Quantum optical interference effects, either one photon or two photon, are thus of fundamental importance [8], as well as providing paths to technological applications [9].

A further test of special relativity, moving towards the domain and ideas of general relativity, is possible in situations where linear acceleration or rotational motion is present [10]. A notable example is the classical Sagnac experiment, where an interferometer is placed on a rotating platform [11-13]. More recent experiments include experimental tests of photonic entanglement in accelerated reference frames [14], the demonstration of how to overcome the shot-noise limit using an entanglement-enhanced optical gyroscope [15], the quantum Sagnac interferometer [16], and the extension of HOM interference to rotating platforms [17].

In this article, we theoretically explore noninertial experiments which require both a quantum mechanical as well as a fully relativistic analysis. In particular, we explore the relation between interference, entanglement, and noninertial rotational motion. We first provide a brief overview of the conceptual

\footnotetext{
*m.toros@ucl.ac.uk

†daniele.faccio@glasgow.ac.uk
}

setup and the relevant classical effect, namely, the Sagnac effect (Sec. II). We then give the theoretical description of quantum experiments on a rotating platform by deriving the Hamiltonian on a generic Hilbert space (Sec. III). We apply the theoretical description to study photon-interferometry experiments and show that the developed model recovers the results for the Sagnac effect in the quantum regime [16] as well as for the recent demonstration of photon bunching in a rotating reference frame [17] (Sec. IV). Finally, we propose a modified HOM interferometer which can be readily implemented using currently available technology. In particular, we discover that simply setting the apparatus in rotational motion can change bunching to antibunching and vice versa, i.e., one can reveal and conceal entanglement (Sec. V).

The overall purpose of this work is thus threefold. The first goal is to give a theoretical foundation for quantum interferometry experiments on rotating platforms, both for matter-wave and photon interferometry. The second is to show that there are nontrivial consequences for the manifestation of entanglement already at low rotation speeds which should be taken into account for state-of-the-art sensing. Finally, the third is that photon interferometry can be used to test the validity of the underlying spacetime symmetries in noninertial and genuinely quantum mechanical experiments.

\section{EXPERIMENTAL SETUP}

We consider an experimental platform rotating at angular frequency $\Omega$ depicted in Fig. 1(a). The system is confined to move on a circle of radius $r$ in the equatorial plane normal to the rotation axis. We further suppose there is a corotating medium with refractive index $n$. For the corotating observer, the light propagation speed is $c / n$ in both directions as can be deduced from symmetry considerations. It is also instructive to describe the same experiment from the inertial frame of the laboratory: One can formally map the circular trajectories to straight-line motions as shown in Fig. 1(b) [18]. In this latter 


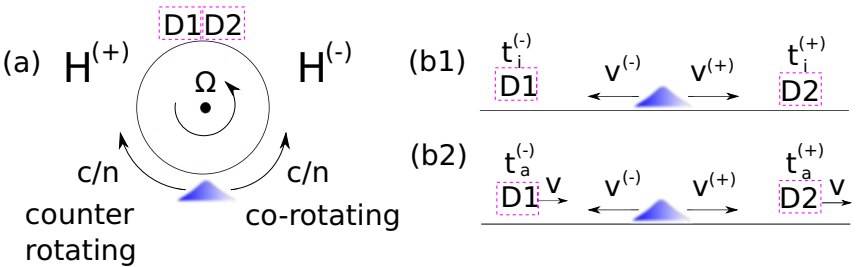

FIG. 1. Conceptual setup. (a) Description from the viewpoint of the corotating observer. The counter-rotating (corotating) quantities are shown on the left (right). The counter-rotating (corotating) Hamiltonians are different, while the light speed in the two directions is equal $(c / n)$. D1 (D2) indicate the detectors for the counterrotating (corotating) direction, respectively. (b) Description from the inertial laboratory frame represented in a straight line. Here, both the Hamiltonian and the speed of light differ in the two directions, i.e., $v^{(-)} \neq v^{(+)}$and $v^{( \pm)} \neq \frac{c}{n}$. (b1) Only the light-drag effect is taken into account; $t_{i}^{(+)}, t_{i}^{(-)}$denote how long it would take to reach the detectors assuming they would not have moved; the subscript $i$ stands for "initial." (b2) Both the light-drag effect and the motion of detectors is taken into account. $t_{a}^{(+)}, t_{a}^{(-)}$denote how long it takes to reach the detectors; the subscript $a$ stands for "actual." $v=r \Omega$ is the speed of the detectors as seen from the inertial laboratory reference frame.

case one has to account for the light-dragging effect [13], i.e., the Fizeau effect.

We now first discuss the relation between the corotating reference frame and the inertial laboratory reference frame, focusing on the classical effects. We start by noting that the speed of light in the corotating reference frame, where the corotating medium is stationary, is the same in the corotating and counter-rotating directions; specifically, we have that the velocities are $\pm \frac{c}{n}$. Using the relativistic velocity addition formula we find the corresponding velocities $u^{( \pm)}$in the inertial laboratory frame,

$$
u^{( \pm)}=\frac{ \pm \frac{c}{n}+v}{1 \pm \frac{c}{n} \frac{v}{c^{2}}} \approx \pm \frac{c}{n}+\alpha v,
$$

where $v=r \Omega$, and $\alpha=1-\frac{1}{n^{2}}$. The corresponding speeds are given by $v^{( \pm)} \approx \frac{c}{n} \pm \alpha v$. See Fig. 1(b) for a graphical illustration of this light-dragging effect, which coincides with the Fizeau effect, but could in principle differ [13].

We assume an initial spatial distance $L$ between the systems and the detectors. We can convert $L$ into a time distance, i.e., the initial time distance [see Fig. 1(b1)], which is given by $t_{i}^{( \pm)}=\frac{L}{v^{( \pm)}}$. Furthermore, exploiting Eq. (1), we find

$$
t_{i}^{( \pm)} \approx \frac{L}{c} n \mp \frac{v L}{c^{2}} \alpha n^{2} .
$$

However, this is different from the actual time it takes the signals to reach the detectors [see Fig. 1(b2)]: We need to take into account also the motion of the detectors. In particular, we have the condition $v^{( \pm)} t_{a}^{( \pm)}=L \pm v t_{a}^{( \pm)}$, which after some algebra readily gives

$$
t_{a}^{( \pm)}=\frac{L}{v^{( \pm)} \mp v} \approx \frac{L}{c} n \pm \frac{L v}{c^{2}} .
$$

In this way we immediately recover the classic Sagnac delay given by $t_{s}=t_{a}^{(+)}-t_{a}^{(-)}=\frac{2 L v}{c^{2}}$. Specifically, to find the usual expression of the Sagnac delay, we set $L=2 \pi r N$, where $N$ denotes the winding number, and define the encircled area as $A=N \pi r^{2}$. Using $v=r \Omega=r 2 \pi f$, we then immediately find $[10,13]$

$$
t_{s}=\frac{8 \pi A f}{c^{2}}
$$

From this discussion it is clear why the description in the corotating reference frame is slightly more convenient: There, only the noninertial motion of the detectors has to be taken into account (through the Hamiltonians). On the other hand, in the laboratory inertial reference frame, one has to account for the motion of the detectors as well as of the medium (again through the Hamiltonians). In short, the advantage of the corotating reference frame is the absence of the light-dragging effect.

Finally, we note that the Sagnac delay can be obtained also from the perspective of the corotating observer where it arises from clock desynchronization [10].

\section{DERIVATION OF THE HAMILTONIAN}

In this section we derive the Hamiltonian for the experiments depicted in Fig. 1(a): We consider a rotating platform, which spins at angular frequency $\Omega$, and we restrict to the dynamics on a circle of radius $r$, centered on the symmetry axis of the rotating platform. Specifically, we will adopt the methods of representation theory [19] and symplectic Hamiltonian mechanics [20] to map the time-evolution generator of the Poincaré algebra to a Hilbert space operator. One could of course make an ad hoc quantization in a noninertial reference frame, but the results might be inconsistent with basic symmetry requirements. Anyhow, we choose the former method which constructs the Hamiltonian starting from basic symmetry considerations of the Poincaré group. As argued in Sec. II, we will for convenience describe the experiment in the corotating reference frame.

One typically starts describing the experiments by setting up a chart, e.g., a Cartesian chart. We note that the chosen spacetime coordinates critically reflect the motion of the observer which affects the resulting description of the dynamics. For example, two observers moving with different speeds or accelerations will use different charts, and hence ascribe different energies to the same system, and hence care must be taken with the choice of the coordinate system. In the following, we will assume that the detectors are stationary in the observer's chart: With this choice there is a simple relation between observables in the description and the quantities measured by the detectors.

We start by recalling the quantization procedure in an inertial reference frame, i.e., with $\Omega=0$. In our case, we will use the polar chart for the laboratory inertial reference frame. Specifically, the line element in an inertial reference frame expressed in the polar chart is given by

$$
d s^{2}=c^{2} d t^{2}-r^{2} d \phi^{2},
$$

where $r$ is a constant in our case (we have restricted the analysis to a $1+1$ spacetime). From the line element in Eq. (5) it is then possible to immediately read the time-evolution Killing vector $\left(\frac{1}{c} \partial_{t}\right)^{\mu}$ [21]. The time-evolution Killing vector (more 
precisely, a vector field) is the tangent vector to the flow lines of a system in free motion. Using the comoment map we can then map the time-evolution Killing vector to the Hilbert space Hamiltonian [19,20],

$$
\partial_{t} \rightarrow \hat{H}
$$

where $\hat{H}$ is the representation of the time-evolution generator on the considered Hilbert space. For example, for a single mode $\hat{a}$ we would have $\hat{H}=\hbar \omega \hat{a}^{\dagger} \hat{a}$, where $\omega$ is the frequency of the oscillator.

To apply the quantization procedure in a noninertial reference frame, i.e., with $\Omega \neq 0$, we have to make an additional step: We have to relate the corotating reference frame to the laboratory inertial reference frame. The reason is simple: We do not know how to directly quantize in a noninertial reference frame, but only in the inertial reference frame. We denote the laboratory (polar) and the corotating (Born) coordinates by the unprimed $\left[x^{\mu}=(c t, r \phi)\right]$ and primed $\left[x^{\mu^{\prime}}=\left(c t^{\prime}, r \phi^{\prime}\right)\right]$ labels, respectively. In particular, we have the following relation [22],

$$
\begin{gathered}
d t=d t^{\prime}, \\
d \phi=d \phi^{\prime}+\Omega d t^{\prime} .
\end{gathered}
$$

It is straightforward to find the corresponding transformation matrix,

$$
\frac{\partial x^{\mu}}{\partial x^{\mu^{\prime}}}=\left[\begin{array}{cc}
1 & 0 \\
\frac{r \Omega}{c} & 1
\end{array}\right],
$$

and to express the Minkowski metric in the two coordinates systems, i.e.,

$$
\begin{aligned}
d s^{2} & =c^{2} d t^{2}-r^{2} d \phi^{2} \\
& =c^{2}\left(1-\frac{\Omega^{2} r^{2}}{c^{2}}\right) d t^{\prime 2}-2 \Omega r^{2} d t^{\prime} d \phi^{\prime}-r^{2} d \phi^{\prime 2} .
\end{aligned}
$$

From Eqs. (10) and (11) we can immediately find the relevant Killing vectors $\left(\frac{1}{c} \partial_{t^{\prime}}\right)^{\mu^{\prime}}=(1,0)^{\top},\left(\frac{1}{c} \partial_{t}\right)^{\mu}=(1,0)^{\top}$, and $\left(\frac{1}{r} \partial_{\phi}\right)^{\mu}=(0,1)^{\top}$. Using Eq. (9) we can then express $\left(\frac{1}{c} \partial_{t^{\prime}}\right)^{\mu^{\prime}}$ in the laboratory coordinates, i.e.,

$$
\left(\frac{1}{c} \partial_{t^{\prime}}\right)^{\mu}=\frac{\partial x^{\mu}}{\partial x^{\mu^{\prime}}}\left(\frac{1}{c} \partial_{t^{\prime}}\right)^{\mu^{\prime}},
$$

which gives $\left(\frac{1}{c} \partial_{t^{\prime}}\right)^{\mu}=\left(1, \frac{r \omega}{c}\right)^{\top}$, and thus

$$
\partial_{t^{\prime}}=\partial_{t}+r \Omega \frac{1}{r} \partial_{\phi} .
$$

We have now expressed the time-evolution Killing vector $\partial_{t^{\prime}}$, which generates the dynamics in the corotating reference frame, in terms of the Killing vectors $\partial_{t}$ and $\frac{1}{r} \partial_{\phi}$, which generate time evolution and space translation in the inertial laboratory reference frame, respectively. We can now map the latter Killing vectors to operators on a Hilbert space using the usual prescription,

$$
\begin{gathered}
\partial_{t} \rightarrow \hat{H}, \\
\frac{1}{r} \partial_{\phi} \rightarrow \hat{P} .
\end{gathered}
$$

Exploiting Eqs. (13)-(15), we can now finally write the timeevolution operator,

$$
\hat{H}_{\mathrm{Born}}=\hat{H}+r \Omega \hat{P}
$$

which we name the Born Hamiltonian. Note that Eq. (16) captures the idea that the dynamics on a rotating platform can be fully explained in terms of the noninertial motion of the detector [18]: The term $\sim \hat{P}$ keeps track of the noninertial motion of the detector. $\hat{P}$, which is the generator of translations, changes the relative distance between the detector and the system, e.g., in a time $\delta t$ the relative distance changes by $r \Omega \delta t$.

We note that the transformation in Eq. (7) leaves the time coordinate unchanged. This can be seen as a Galilean-type transformation on a circle, which we now generalize to a Lorentz-type transformation. In particular, we consider

$$
\begin{gathered}
d t=\Gamma d t^{\prime}+A \Gamma \frac{r^{2} \Omega}{c^{2}} d \phi^{\prime}, \\
d \phi=B \Gamma d \phi^{\prime}+\Gamma \Omega d t^{\prime},
\end{gathered}
$$

where $\Gamma=\left[1-\left(\frac{\Omega r}{c}\right)^{2}\right]^{-\frac{1}{2}}$. If we set $A=1$ and $B=1$, the transformation is formally equivalent to a Lorentz boost with speed $v=r \Omega$, while if we set $A=0$ and $B=\Gamma^{-1}$, we obtain the transformation considered by Post [13]. We find that the Hamiltonian is insensitive to the value of $A$, but depends on the chosen value of $B$. In the following, we set $B=1$, which leads to the Hamiltonian

$$
\hat{H}_{\mathrm{Born}}^{\mathrm{rel}}=\Gamma(\hat{H}+r \Omega \hat{P}) .
$$

Equation (19) can be seen as a relativistic Born Hamiltonian, which generalizes Eq. (16).

We can also analyze nonuniform rotations using the above formalism by considering a time-dependent angular frequency $\Omega_{t}$ (we remark that the time-evolution vector does not need to be generally a Killing vector). We repeat the derivation in this section with the formal replacements

$$
\begin{gathered}
\Omega \rightarrow \Omega_{t}, \\
\Gamma \rightarrow \Gamma_{t}=\left[1-\left(\frac{\Omega_{t} r}{c}\right)^{2}\right]^{-\frac{1}{2}} .
\end{gathered}
$$

At the end we obtain in place of Eq. (19) the following Hamiltonian,

$$
\hat{H}_{\mathrm{Born}}^{\mathrm{rel}}(t)=\Gamma_{t}\left(\hat{H}+r \Omega_{t} \hat{P}\right) .
$$

In this case one expects two physical effects, one related to the (geometrical) Sagnac phase, and a possible new contribution related to a dynamical phase, which typically emerges in situations where there is a time dependence in the Hamiltonian.

\section{PHOTON-INTERFEROMETRY EXPERIMENTS}

We now apply the Hamiltonian in Eq. (19) to photon interferometry. We use the Abraham relation between kinetic momentum and energy [23,24],

$$
\hat{H}=n c|\hat{P}| \text {. }
$$


We combine Eqs. (19) and (23) to find

$$
\hat{H}_{\text {Born }}^{( \pm)}=\Gamma(1 \pm \beta) \hat{H}
$$

where the positive (negative) superscript denotes the counterrotating (corotating) motion to be measured by the detector D1 (D2), and $\beta=\frac{r \Omega}{n c}$. We note that Eq. (24) suggests a simple physical interpretation of the Hamiltonian as the relativistic Doppler-shifted energy of the system.

We can finally write the total Hamiltonian of the system,

$$
\hat{H}_{\text {Born }}=\hat{H}_{\text {Born }}^{(+)} \otimes \mathbb{I}+\mathbb{I} \otimes \hat{H}_{\text {Born }}^{(-)},
$$

where we have assumed that the photons moving in opposite directions do not interact, and $\mathbb{I}$ denotes the identity operators. In summary, the total Hilbert space can be written as $\mathcal{H}=$ $\mathcal{H}^{(+)} \otimes \mathcal{H}^{(-)}$, where $\mathcal{H}^{(+)}\left(\mathcal{H}^{(-)}\right)$denotes the Hilbert space of the counter-rotating (corotating) modes.

The time parameter $t$ that keeps track of the dynamics through Schrödinger's equation is ticking as a clock following the detectors' motion; this is a direct consequence of the quantization procedure that leads to the Hamiltonian in Eq. (25). However, here we are mainly interested in the dominant effects where one can approximate the Lorentz factor as $\Gamma \sim 1$ and the distances and times coincide to those measured by a ruler and a clock in the inertial laboratory frame.

We now further develop the model by adopting Glauber's theory of photodetection [25]. Here, we will focus on the experimental situation of photons with a coherence time that is short compared to the time resolution of the detectors, but temporal aspects could be easily taken into account [26,27]. In other words, here we are not concerned about when the detectors click, but only which detector clicks. We assume that in the detectors' time window only one photon or one photon pair has been measured.

To analyze photon-interferometry experiments we will work in the Schrödinger picture [28], where we will denote the initial (final) state with the subscripts $i(f)$. We consider the experimental situation where at time $t_{i}=0$ the photon is prepared in a state $\left|\psi_{i}\right\rangle$, and then constrained to move in a circular motion for a time $t_{f}=\frac{L}{c} n$ resulting in a state $\left|\psi_{f}\right\rangle$, where $L$ is the traveled distance. Although one can always postulate a given initial state, it is nonetheless instructive to compare the state $\left|\psi_{i}\right\rangle$, which is assumed to be generated by the apparatus corotating with the platform, with the state generated by the same apparatus when the platform is not rotating, i.e., when $\Omega=0$. In particular, it is reasonable to assume that the frequencies of the initial states generated in the two experimental situations differ by at most $\sim \frac{\Omega}{2 \pi}$. However, such a difference in the initial state produces only subleading effects which are not amplified during time evolution, as can be explicitly verified using the formulas we will develop. One can thus approximate the initial states generated on the rotating platform with the states that would be generated at $\Omega=0$.

The time evolution is given by the usual Schrödinger equation with the Hamiltonian in Eq. (25), i.e.,

$$
\hat{H}^{(2)}=\hbar \int d \omega\left[\omega^{(+)} \hat{a}^{\dagger}(\omega) \hat{a}(\omega)+\omega^{(-)} \hat{b}^{\dagger}(\omega) \hat{b}(\omega)\right],
$$

where we have defined $\omega^{( \pm)}=(1 \pm \beta) \omega$, and $\hat{a}(\hat{b})$ is the counter (corotating) mode.
The state $\left|\psi_{f}\right\rangle$ then interferes at a beam splitter and one measures the outputs using two detectors: The input modes are $\hat{a}$ and $\hat{b}$ and we denote the output modes by $\hat{c}$ and $\hat{d}$. Here, we consider the following relation between the input and output modes,

$$
\left[\begin{array}{l}
\hat{c}(\omega) \\
\hat{d}(\omega)
\end{array}\right]=\frac{1}{\sqrt{2}}\left[\begin{array}{cc}
1 & 1 \\
1 & -1
\end{array}\right]\left[\begin{array}{l}
\hat{a}(\omega) \\
\hat{b}(\omega)
\end{array}\right] .
$$

In particular, we are interested in the probability of detecting photons in the modes $\hat{c}$ or $\hat{d}$. To this end, it is convenient to define the temporal modes [29],

$$
\hat{c}(t)=\mathcal{F}_{t}[\hat{c}(\omega)], \quad \hat{d}(t)=\mathcal{F}_{t}[\hat{d}(\omega)],
$$

where $\mathcal{F}_{t}[\cdot]=\frac{1}{\sqrt{2 \pi}} \int d \omega \cdot e^{-i \omega t}$. In particular, we define the single-photon probability of detection as

$$
P_{c}^{(1)}=\int d t\left\langle\psi_{f}\left|\hat{c}^{\dagger}(t) \hat{c}(t)\right| \psi_{f}\right\rangle
$$

with a similar definition for the probability $P_{d}^{(1)}$ for the output mode $\hat{d}$. In addition, we also define the two-photon probability of detection,

$$
P^{(2)}=\int d t_{1} \int d t_{2}\left\langle\psi_{f}\left|\hat{d}^{\dagger}\left(t_{1}\right) \hat{c}^{\dagger}\left(t_{2}\right) \hat{c}\left(t_{2}\right) \hat{d}\left(t_{1}\right)\right| \psi_{f}\right\rangle,
$$

which gives the coincidence probability. For the case $P^{(2)}<$ 0.5 we speak of coalescence or HOM photon bunching and for $P^{(2)}>0.5$ we speak of photon anticoalescence or antibunching. Classically, one is limited to values $0.25<P^{(2)}<0.5$, making coincidence probabilities a valuable tool to assess the quantum nature of the electromagnetic field. Importantly, antisymmetrization and photon anticoalescence reveals hidden entanglement, as has already been demonstrated experimentally in a nonrotating setup [30].

We consider first the experimental situation with a generic single-photon input state,

$$
\left|\psi_{f}\right\rangle=\int d \omega\left[\psi_{a, f}(\omega) \hat{a}^{\dagger}(\omega)+\psi_{b, f}(\omega) \hat{b}^{\dagger}(\omega)\right]|0\rangle,
$$

where $\psi_{a, f}(\omega), \psi_{b, f}(\omega)$ are one-photon wave functions. From Eqs. (29) and (31), exploiting Eqs. (27) and (28), we find

$$
P_{c, d}^{(1)}=\frac{1}{2} \pm \frac{1}{2} \int d \omega\left[\psi_{a, f}^{*}(\omega) \psi_{b, f}(\omega)+\text { c.c. }\right],
$$

where we have imposed the normalization of the state, i.e., $\left\langle\psi_{f} \mid \psi_{f}\right\rangle=1$. As an example let us consider the quantum Sagnac experiment [16]: A photon is prepared in a superposition of counterpropagating modes before interfering at the beam splitter [see Fig. 2(a)]. Specifically, we consider the initial state in Eq. (31) with the one-photon wave functions $\psi_{a, i}(\omega)=\psi_{b, i}(\omega)=g(\omega)$, where $g(\omega)$ is a Gaussian with mean frequency $\mu$ and bandwidth $\sigma$. After the time evolution using Eq. (26) we have the state in Eq. (31) with $\psi_{a, f}(\omega)=$ $g(\omega) e^{i \omega \beta t_{f}}$ and $\psi_{b, f}(\omega)=g(\omega) e^{-i \omega \beta t_{f}}$. Using Eq. (32) we find the single-photon detection probability,

$$
P_{c, d}^{(1)}=\frac{1}{2}\left[1 \pm e^{-\left(\frac{\sigma t_{s}}{2}\right)^{2}} \cos \left(\mu t_{s}\right)\right]
$$

where $t_{s}$ is the classical Sagnac delay introduced in Eq. (4). If we now make the further approximation $|g(\omega)|^{2} \sim \delta(\mu-\omega)$, 

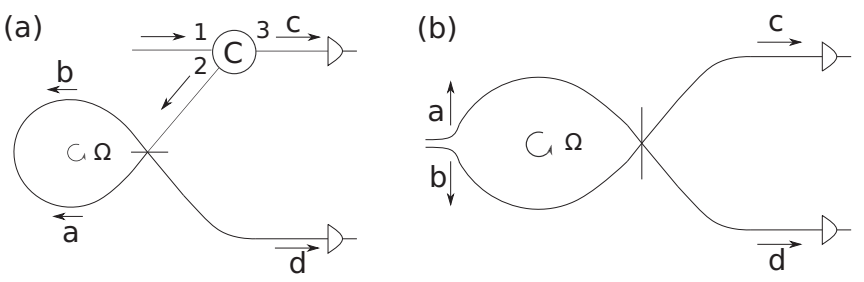

FIG. 2. (a) Quantum Sagnac experiment [16]. The element $C$ denotes the circulator which allows only the paths 1 to 2 and 2 to 3. A photon enters through the path 1 , is directed into path 2 , and then interferes for the first time with the beam splitter. After evolving in counterprogating directions, the photon then interferes again at the beam splitter, after which it is detected. (b) Hong-Ou-Mandel experiment on a rotating platform [17]. Two identical photons counterpropagate before interfering at a beam splitter. One detects the arrival of the photons and extracts the coincidence probability $P^{(2)}$. In both cases, the setups are placed on a rotating platform.

i.e., $\sigma \rightarrow 0$, we then recover the results reported in Ref. [16], i.e., $P_{c, d}^{(1)}=\frac{1}{2}\left[1 \pm \cos \left(\mu t_{s}\right)\right]$.

We next consider the two-photon state,

$$
\left|\psi_{f}\right\rangle=\int d \omega_{1} \int d \omega_{2} \psi_{f}\left(\omega_{1}, \omega_{2}\right) \hat{a}^{\dagger}\left(\omega_{1}\right) \hat{b}^{\dagger}\left(\omega_{2}\right)|0\rangle,
$$

where $\psi_{f}\left(\omega_{1}, \omega_{2}\right)$ is the two-photon spectrum. From Eqs. (30) and (34), exploiting Eqs. (27) and (28), we find

$$
P^{(2)}=\frac{1}{2}-\frac{1}{2} \int d \omega_{1} \int d \omega_{2} \psi_{f}^{*}\left(\omega_{1}, \omega_{2}\right) \psi_{f}\left(\omega_{2}, \omega_{1}\right),
$$

where we have imposed the normalization $\left\langle\psi_{f} \mid \psi_{f}\right\rangle=1$. As an example we consider the Hong-Ou-Mandel experiment on a rotating platform [17]: Two identical photons counterpropagate before interfering at a beam splitter [see Fig. 2(b)]. The experimentalist controls the initial time delay $\delta t$ of the mode $\hat{a}$; the initial state is given by Eq. (34) with the twophoton spectrum $\psi_{i}\left(\omega_{1}, \omega_{2}\right)=g\left(\omega_{1}\right) g\left(\omega_{2}\right) e^{-i \omega_{1} \delta t}$. After the time evolution we find the final state in Eq. (34) with

$$
\psi_{f}\left(\omega_{1}, \omega_{2}\right)=g\left(\omega_{1}\right) g\left(\omega_{2}\right) e^{-i \omega_{1} \delta t} e^{i \beta\left(\omega_{1}-\omega_{2}\right)} .
$$

Using Eq. (35) we then immediately find the coincidence probability,

$$
P^{(2)}=\frac{1}{2}-\frac{1}{2} e^{-\sigma^{2}\left(t_{s}-\delta t\right)^{2}},
$$

where $t_{s}$ is the classical Sagnac delay.

Above, we have considered identical photons with a separable spectrum [7], but one could also consider identical frequency-entangled photons. For example, if we consider spontaneous parametric down-conversion (SPDC) type-I twophoton generation [31], we again find the coincidence probability in Eq. (37). It would thus seem that entanglement in combination with rotational motion leaves no trace on the photon coincidence rate $P^{(2)}$ measurement. We now further explore this question.

\section{MANIFESTATION OF ENTANGLEMENT THROUGH ROTATION}

Entanglement can manifest itself in a HOM coincidence rate measurement through anticoalescence, i.e., $P^{(2)}>0.5$.

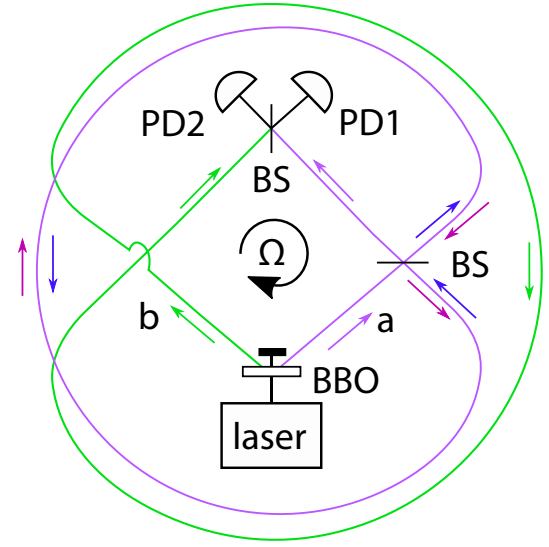

FIG. 3. Layout of proposed quantum Sagnac/Hong-Ou-Mandel interferometer on a rotating platform. Two entangled photons are emitted from the BBO crystal: Photon a (purple arrow) enters a Sagnac interferometer using the lower $50 / 50$ beam splitter (BS) and exits towards the upper 50/50 BS where two-photon HOM interference occurs with photon $\mathrm{b}$ (green arrow) that circles around the setup (in order to maintain the same overall path length as photon a). Coincidence counts are measured between detectors PD1 and PD2 as a function of the rotation frequency $\Omega$.

In particular, for a completely antisymmetric spectrum, i.e., $\psi\left(\omega_{1}, \omega_{2}\right)=-\psi\left(\omega_{2}, \omega_{1}\right)$, one obtains perfect anticoalescence, but even with a partially antisymmetric spectrum one can have $P^{(2)}>0.5$, thus witnessing entanglement. As we show below, this manifestation of entanglement may be susceptible to the motion of the interferometer.

We consider the experimental setup depicted in Fig. 3. As an initial state we consider a SPDC type-I two-photon state (i.e., two photons with the same polarization) with the spectrum

$$
\psi_{i}\left(\omega_{1}, \omega_{2}\right)=\delta\left(\omega_{1}+\omega_{2}-2 \mu\right) g\left(\omega_{1}\right) g\left(\omega_{2}\right),
$$

where we have omitted the normalization. We note that the initial spectrum in Eq. (38) is completely symmetric, i.e., $\psi\left(\omega_{1}, \omega_{2}\right)=\psi\left(\omega_{2}, \omega_{1}\right)$, and gives $P^{(2)}=0$ at $\Omega=0$.

We now consider the same setup with the interferometer in a constant rotational motion with frequency $\Omega \neq 0$. The final spectrum of the two-photon state changes to

$$
\psi_{f}\left(\omega_{1}, \omega_{2}\right)=\psi_{i}\left(\omega_{1}, \omega_{2}\right) \cos \left(\beta \omega_{1} t_{f}\right) e^{-i \beta \omega_{2} t_{f}},
$$

where the factor $\cos \left(\beta \omega_{1} t_{f}\right)$ results from the interference of the mode $\hat{a}$; by "final state" we again mean the state that arrives at the last beam splitter. Using Eq. (35) we then find the coincidence probability,

$$
P^{(2)}=\frac{1}{2}-\frac{\cos \left(\mu t_{s}\right) e^{-\frac{1}{8} \sigma^{2} t_{s}^{2}}+\frac{1}{2}\left(1+e^{-\frac{1}{2} \sigma^{2} t_{s}^{2}}\right)}{2\left[1+\cos \left(\mu t_{s}\right) e^{-\frac{1}{8} \sigma^{2} t_{s}^{2}}\right]} .
$$




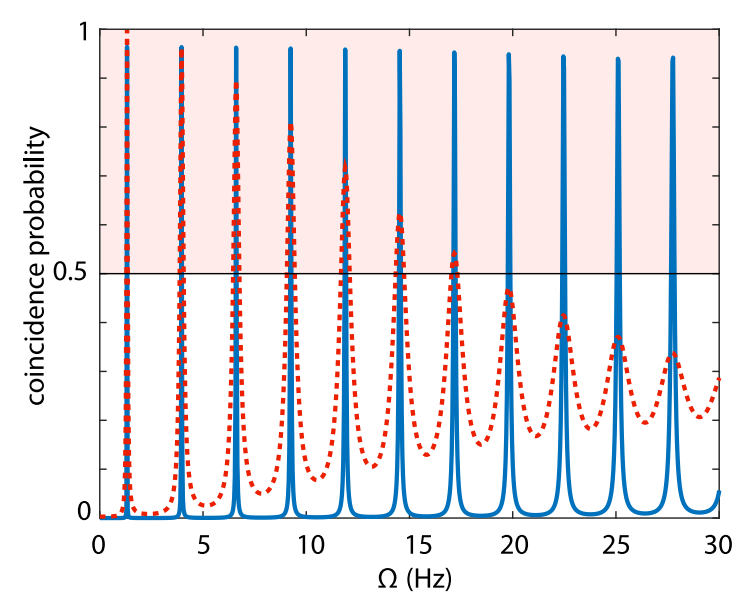

FIG. 4. Coincidence plot as a function of the angular frequency $\Omega=2 \pi f$. We have set the interferometer area $A=22.7 \mathrm{~m}^{2}$, $\mu=2.36 \times 10^{15} \mathrm{~Hz}$, corresponding to a typical photon carrier wavelength of $800 \mathrm{~nm}$. Two curves are shown for two different bandwidths, $\sigma=1.47 \times 10^{13} \mathrm{~Hz}$ (blue solid curve) and $\sigma=$ $1.18 \times 10^{14} \mathrm{~Hz}$ (dashed red curve), corresponding to 5- and 40$\mathrm{nm}$ bandwidths, respectively. The shaded region corresponding to $P^{(2)}>0.5$ indicates the region where measurements imply photon entanglement.

We have plotted $P^{(2)}$ as a function of the angular frequency $\Omega$ in Fig. 4 with the interferometer area $A=22.7 \mathrm{~m}^{2}$ (assuming the photons travel through a 100-m-long fiber, wound 35 times along a 0.9-m-diameter loop) and $\mu=$ $2.36 \times 10^{15} \mathrm{~Hz}$, corresponding to a typical photon carrier wavelength of $800 \mathrm{~nm}$. Two curves are shown for two different photon bandwidths, $\sigma=1.47 \times 10^{13} \mathrm{~Hz}$ (blue solid curve) and $\sigma=1.18 \times 10^{14} \mathrm{~Hz}$ (dashed red curve), corresponding to 5 and $40 \mathrm{~nm}$, respectively. The shaded region corresponding to $P^{(2)}>0.5$ indicates the presence of entanglement that manifests as photon anticoalescence. Short bandwidths, i.e., long coherence photons, show a periodic series of revivals of entanglement with increasing rotation frequency. For larger photon bandwidths, i.e., shorter coherence lengths, increasing the relative photon delay by increasing the rotation speed leads to a reduction of the coincidence peak values and of the overall fringe visibility. This is a result of the loss of mutual coherence between the two interfering photons.

The proposal shown in Fig. 3 can be also seen as a combination of the setups shown in Fig. 2, which can be exploited to gain an intuitive understanding of the results. As in the case of the quantum Sagnac and the HOM setup, also in this case the wave packets traveling in the corotating and counter-rotating directions acquire a relative time delay during time evolution which is mathematically represented by a phase factor. Specifically, the paths denoted by the purple and blue arrows in Fig. 3 can be related to the quantum Sagnac and contribute an interference factor $e^{-i \beta \omega_{1} t_{f}}+e^{+i \beta \omega_{1} t_{f}} \sim \cos \left(\beta \omega_{1} t_{f}\right)$. Together these paths, i.e., the purple and blue arrows, form half of the HOM setup, while the other half is depicted by the corotating green path which in turn contributes a time delay $e^{-i \beta \omega_{2} t_{f}}$. For the whole setup we thus find a factor $\cos \left(\beta \omega_{1} t_{f}\right) e^{-i \beta \omega_{2} t_{f}}$ which is not symmetric under exchange of the frequencies, i.e., $\omega_{1} \leftrightarrow \omega_{2}$. In frequency space these time delays then change the final biphoton spectrum from symmetric to antisymmetric and vice versa, resulting in oscillations between bunching and antibunching, respectively, as seen in Fig. 4. The paths $a$ and $b$ in Fig. 3 forming the asymmetric HOM setup can be also seen as reminiscent of an unbalanced Mach-Zehnder setup [32].

The asymptotic value for the coincidence probability in Fig. 4 can also be intuitively understood in terms of quantum Sagnac and the HOM setups. At high rotation frequency the counterpropagating mode (blue arrow) no longer interferes with the corotating modes (purple and green arrows) due to nonoverlapping frequency spectra. Loosely speaking, the counterpropagating mode (blue arrow) can be associated with half of the initial mode $\hat{a}$, while the other half of the mode $\hat{a}$ corotates (purple arrow) and interferes with the other corotating mode $\hat{b}$ (green arrow). From Eq. (40) we find at high rotation frequency the value $P^{(2)} \sim 1 / 4$; this is halfway between a full dip, $P^{(2)} \sim 0$, and the case without bunching or antibunching, $P^{(2)} \sim 1 / 2$. As the frequency spectrum of the two corotating modes is identical (blue and green arrow), i.e., no time delay exists between them, they bunch together at the output ports of the beam splitter. Hence the counterrotating (corotating) part of the mode $\hat{a}$ is associated with $P^{(2)} \sim 1 / 2\left(P^{(2)} \sim 0\right)$. The coincidence probability at high rotation frequency can be thus seen as the average behavior, i.e., $P^{(2)} \sim(0+1 / 2) / 2=1 / 4$.

The experimental setups shown in Fig. 2 and the one shown in Fig. 3 are substantially different, both experimentally and conceptually. In particular, the HOM on a rotating platform [17] is a symmetric setup, which is unable to induce the antibunching behavior of photons, but produces only a coincidence dip. Although photon bunching below $P^{(2)}<\frac{1}{4}$ implies quantum interference phenomena, one cannot assert anything about entanglement. On the other hand, the setup in Fig. 3 is asymmetric, and as shown in Fig. 4 is able to invert a coincidence dip, i.e., $P^{(2)}<\frac{1}{2}$, to a spike, i.e., $P^{(2)}>\frac{1}{2}$. Its importance lies in that photon antibunching $P^{(2)}>\frac{1}{2}$ implies entanglement.

The antisymmetrization of the photon spectrum, which leads to a modification of the coincidence probability, is a direct consequence of the noninertial motion of the platform. More generally, this shows that rotational motion can activate dormant asymmetries in the experimental setup leading to an antisymmetric spectrum. It is also interesting to consider an initial antisymmetric spectrum $\psi_{i}$; the proposed experiment shows that $\psi_{i}$ can become symmetrized during time evolution, fully concealing the anticoalescence signature of entanglement.

These effects can be traced to the impossibility of clock synchronization along a closed loop on the rotating platform. In particular, the Hamiltonian in Eq. (26) can be linked to the effect of clock desynchronization [10]. It is important to note that this is a genuine relativistic effect, which is not expected to arise in a Newtonian theory, although it imprints a non-negligible experimental trace in the regime typically associated with the latter. This is different from the observerdependent entanglement effect in noninertial reference frames [33], expected to arise as a consequence of the Unruh radiation $[34,35]$ which vanishes at low accelerations. 


\section{CONCLUSIONS}

We have developed a formalism for describing quantum experiments on rotating platforms, both matter-wave and photon interferometry, exploiting the map between symmetry generators and Hilbert space operators. The dynamics assumes a simple form with the time evolution given by the Born Hamiltonian. The developed theory can be represented on a generic Hilbert space and is valid in regimes where the particle number is conserved, as is the case of a typical table-top laboratory experiment.

We have further developed the theory to analyze two recent photon-interferometry experiments, namely, the quantum Sagnac and the HOM experiment on a rotating platform. We have also proposed a modified HOM interferometer where entanglement can be revealed or concealed depending on the rotational frequency. Specifically, we have shown that rotations together with an asymmetry of the experimental setup can strongly affect the bunching and antibunching behavior, and hence the manifestation of entanglement.

The interferometer shown in Fig. 3 can generate both bunching as well as antibunching solely through rotation, a feature which distinguishes it from the previously considered HOM interferometer on a rotating platform [17]. Furthermore, the strength of the effect at low rotational speed is suggestive that such phenomena are all but rare. Indeed, we note that asymmetries in the setups of complex experiments as well as rotations, both controlled and spurious, are ubiquitous. It is thus likely that these effects will have ramifications for quantum-sensing applications.

The proposed experiment can be also viewed as a test of the underlying Poincaré symmetry group for entangled system. The proposal is based on a theoretical model that relies heavily on intrinsic features of both relativity and quantum mechanics. Although we do not suspect deviations from the predicted behavior, we nonetheless note that entanglement in noninertial reference frames has not yet been fully experimentally scrutinized. We conclude by recalling that classical tests of spacetime symmetries such as the Michelson-Morley and the Sagnac experiment were also initially devised with the goal of reaffirming the established theories, but ended profoundly affecting the elementary notions of space and time.

\section{ACKNOWLEDGMENT}

The authors acknowledge support from the EU H2020FET project TEQ (Grant No. 766900), from the EPSRC (U.K., Grant No. EP/M009122/1), and from the European Union's Horizon 2020 research and innovation programme under Grant Agreement No. 820392.
[1] H. Minkowski, Raum und zeit, Jahresber. Dtsch. Math. Ver. 18, 75 (1909).

[2] Minkowski Spacetime: A Hundred Years Later, edited by V. Petkov, Vol. 6 (Springer, Berlin, 2010).

[3] W. A. Von Ignatowsky, Einige allgemeine Bemerkungen zum Relativitätsprinzip, Verh. Dtsch. Phys. Ges. Berlin 12, 788 (1910).

[4] S. Liberati, Tests of Lorentz invariance: A 2013 update, Classical Quantum Gravity 30, 133001 (2013).

[5] H. P. Robertson, Postulate versus observation in the special theory of relativity, Rev. Mod. Phys. 21, 378 (1949).

[6] A. A. Michelson and E. W. Morley, On the relative motion of the earth and of the luminiferous ether, Sidereal Messenger 6, 306 (1887).

[7] C.-K. Hong, Z.-Y. Ou, and L. Mandel, Measurement of Subpicosecond Time Intervals between Two Photons by Interference, Phys. Rev. Lett. 59, 2044 (1987).

[8] L. Mandel, Quantum effects in one-photon and two-photon interference, in More Things in Heaven and Earth (Springer, Berlin, 1999), pp. 460-473.

[9] A. Lyons, G. C. Knee, E. Bolduc, T. Roger, J. Leach, E. M. Gauger, and D. Faccio, Attosecond-resolution Hong-Ou-Mandel interferometry, Sci. Adv. 4, eaap9416 (2018).

[10] É. Gourgoulhon, Special Relativity in General Frames (Springer, Berlin, 2016).

[11] G. Sagnac, L'éther lumineux démontré par l'effet du vent relatif d'éther dans un interféromètre en rotation uniforme, C. R. Acad. Sci. 157, 708 (1913).

[12] G. Sagnac, Sur la preuve de la réalité de l'éther lumineux par l'expérience de l'interférographe tournant, C. R. Acad. Sci. 157, 1410 (1913).
[13] E. J. Post, Sagnac effect, Rev. Mod. Phys. 39, 475 (1967).

[14] M. Fink, A. Rodriguez-Aramendia, J. Handsteiner, A. Ziarkash, F. Steinlechner, T. Scheidl, I. Fuentes, J. Pienaar, T. C. Ralph, and R. Ursin, Experimental test of photonic entanglement in accelerated reference frames, Nat. Commun. 8, 15304 (2017).

[15] M. Fink, F. Steinlechner, J. Handsteiner, J. P. Dowling, T. Scheidl, and R. Ursin, Entanglement-enhanced optical gyroscope, New J. Phys. 21, 053010 (2019).

[16] G. Bertocchi, O. Alibart, D. B. Ostrowsky, S. Tanzilli, and P. Baldi, Single-photon Sagnac interferometer, J. Phys. B: At., Mol. Opt. Phys. 39, 1011 (2006).

[17] S. Restuccia, M. Toroš, G. M. Gibson, H. Ulbricht, D. Faccio, and M. J. Padgett, Photon Bunching in a Rotating Reference Frame, Phys. Rev. Lett. 123, 110401 (2019).

[18] A. Tartaglia and M. L. Ruggiero, The Sagnac effect and pure geometry, Am. J. Phys. 83, 427 (2015).

[19] P. Woit, Quantum Theory, Groups and Representations (Springer, Berlin, 2017).

[20] A. Cannas Da Silva and A. Cannas Da Salva, Lectures on Symplectic Geometry, Vol. 3575 (Springer, Berlin, 2001).

[21] E. Poisson, A Relativist's Toolkit: The Mathematics of BlackHole Mechanics (Cambridge University Press, Cambridge, U.K., 2004).

[22] G. Rizzi and M. L. Ruggiero, Space geometry of rotating platforms: An operational approach, Found. Phys. 32, 1525 (2002).

[23] M. J. Padgett, On diffraction within a dielectric medium as an example of the Minkowski formulation of optical momentum, Opt. Express 16, 20864 (2008).

[24] S. M. Barnett, Resolution of the Abraham-Minkowski Dilemma, Phys. Rev. Lett. 104, 070401 (2010). 
[25] R. J. Glauber, The quantum theory of optical coherence, Phys. Rev. 130, 2529 (1963).

[26] T. Legero, T. Wilk, A. Kuhn, and G. Rempe, Characterization of single photons using two-photon interference, Adv. At., Mol., Opt. Phys. 53, 253 (2006).

[27] H. P. Specht, J. Bochmann, M. Mücke, B. Weber, E. Figueroa, D. L. Moehring, and G. Rempe, Phase shaping of single-photon wave packets, Nat. Photonics 3, 469 (2009).

[28] K. Wang, Quantum theory of two-photon wavepacket interference in a beamsplitter, J. Phys. B: At., Mol. Opt. Phys. 39, R293 (2006).

[29] K. J. Blow, R. Loudon, S. J. D. Phoenix, and T. J. Shepherd, Continuum fields in quantum optics, Phys. Rev. A 42, 4102 (1990).

[30] A. Fedrizzi, T. Herbst, M. Aspelmeyer, M. Barbieri, T. Jennewein, and A. Zeilinger, Anti-symmetrization re- veals hidden entanglement, New J. Phys. 11, 103052 (2009).

[31] M. Barbieri, E. Roccia, L. Mancino, M. Sbroscia, I. Gianani, and F. Sciarrino, What Hong-Ou-Mandel interference says on two-photon frequency entanglement, Sci. Rep. 7, 7247 (2017).

[32] D. V. Strekalov, T. B. Pittman, and Y. H. Shih, What we can learn about single photons in a two-photon interference experiment, Phys. Rev. A 57, 567 (1998).

[33] I. Fuentes-Schuller and R. B. Mann, Alice Falls into a Black Hole: Entanglement in Noninertial Frames, Phys. Rev. Lett. 95, 120404 (2005).

[34] P. C. W. Davies, Scalar production in Schwarzschild and Rindler metrics, J. Phys. A: Math. Gen. 8, 609 (1975).

[35] W. G. Unruh, Notes on black-hole evaporation, Phys. Rev. D 14, 870 (1976). 